\title{
The effect of prism strength and response mode on the magnitude of the correction effect in prism viewing
}

\author{
LAWRENCE E. MELAMED and PETER A. BECKETT \\ Kent State University, Kent, Ohio 44240 \\ and \\ BENJAMIN WALLACE \\ Western Illinois University, Macomb, Illinois 61455
}

\begin{abstract}
In the correction effect paradigm, the apparent straight-ahead is judged, under prismatic viewing, in both an illuminated and a darkened room condition. The correction effect occurs to the extent that the straight-ahead is judged more veridically in the illuminated than in the darkened room. In the present experiment, equivalent correction effects were obtained using prism magnitudes from 10 to $30 \mathrm{D}$. Further, correction effects were not obtained when the straight-ahead was measured with a pointing response rather than by the use of a visual positioning task. The implications of these data for explanations of the correction effect that have been presented in the literature are discussed.
\end{abstract}

The correction effect (Rock, Goldberg, \& Mack, 1966) occurs when an observer wearing prisms positions a spot of light more veridically straight-ahead in an illuminated room than in a dark one. As an example of this phenomenon, consider a subject viewing a spot of light through 20 -D laterally displacing prisms. The spot is positioned on a far wall which is perpendicular to the subject's median plane. With base-right prisms, the spot is displaced approximately $11.4^{\circ}$ to the subject's left. In the initial positioning task in the darkened room, in which the subject must place the spot at the apparent straightahead, a typical response would be for the subject to place the spot about $10^{\circ}$ to his or her right. When the room is then illuminated, this subject will now position the spot perhaps $6^{\circ}$ to his or her right under the same instructions as before. The subject has shown a correction effect on the order of $4^{\circ}$; the placement is $4^{\circ}$ more veridical in the latter condition.

Rock et al. found that regardless of whether the prisms displaced the image in the horizontal or in the vertical plane, the subjects were more veridical in their judgments in the illuminated room than in darkness, on the order of $4^{\circ}$, for the 20-D prisms they used. Moreover, if the room lights were left on an additional period after setting the spot straight

This research was supported by Grant MH-20504 from NIMH to the first author. Requests for reprints should be sent to Lawrence E. Melamed, Department of Psychology, Kent State University, Kent, Ohio 44242. Peter Beckett is now at St. Leo College, St. Leo, Florida 33574. ahead, the subjects showed an aftereffect in locating the visual straight-ahead when tested subsequently in the dark with the prisms removed. The aftereffect was in the direction of the displacement produced by the prisms.

Rock et al. were impressed with these findings because none of the traditional tasks to induce adaptation had been performed by the subjects. They simply stared at a far wall. A perceptual interpretation of the aftereffects was offered. Rock et al. argued that the correction effect showed that their subjects had a source of information about their phenomenal location in space relative to their surroundings which, paired with changes in eye position for apparent spatial directions, could serve as a strong determinant of visual adaptation to prismatic displacement.

The proposal that the correction effect indicates a perceptual reorientation that underlies prism adaptation has not been supported by subsequent research. In a series of experiments (Wallace, Melamed, \& Cohen, 1973; Wallace, Melamed, \& Kaplan, 1973), the most important findings concerned the aftereffects. It was demonstrated, and replicated, that aftereffects are obtained in the correction effect paradigm only when the observers are free to align the spot of light to the straight-ahead themselves. Directing the experimenter in its placement will produce a correction effect, but there will be no subsequent aftereffect. Rock et al. had their subjects position the light themselves. Moreover, the aftereffect occurs very rapidly. It is actually present immediately 
after the correction effect measurement itself. The subsequent exposure to the far wall is superfluous. The aftereffect was not found to increase in magnitude following the 10-min exposure to the optically displaced room. The need for active movement of the projected light would support the argument that the projected spot under these circumstances simply represented feedback for arm position and thus presented the opportunity for prism adaptation to occur.

What is unclear from the findings presented above is the extent to which the correction effect and prism adaptation can be considered as phenomena of the same class, i.e., have similar properties. The research reviewed indicates only that the experimental conditions that are sufficient for producing a correction effect do not invariably result in aftereffects. The experimental manipulations involved in the present study have been used in prism adaptation paradigms and have produced consistent effects. It was expected that the data obtained would not only provide further parametric information about the correction effect, but would also serve to clarify its relationship to the process of prism adaptation.

The first part of the present study involved measuring the correction effect for prismatic deviations from 10 to $30 \mathrm{D}$ in 5-D steps. If the correction effect functions in a manner analogous to the visual localization aftereffects of prism adaptation, it should monotonically increase with increases in the magnitude of the imposed prismatic deviation. In the second part of the study, the subjects pointed to the apparent straight-ahead within the correction effect paradigm rather than positioning a spot of light as in the usual procedure. If the correction effect represents an adjustment in spatial coding akin to that which occurs in prism adaptation, the correction effect should occur for the pointing response as well as for the visual positioning task. Such adjustments routinely occur in both response modalities in prism adaptation research (e.g., Wilkinson, 1971).

\section{EXPERIMENT 1}

\section{Method}

\section{Subjects}

Eighty female undergraduates from the introductory psychology sections served as subjects. Only individuals who did not wear corrective glasses were selected.

\footnotetext{
Apparatus

The subject was seated at a small wooden table, $60.8 \times 121.6 \mathrm{~cm}$ with her head securely positioned on a combination head- and chinrest. The subject wore Risley rotating prisms attached to the front surface of a welder's goggles. To the right of the subject, on the table, was a partition, $91.2 \mathrm{~cm}$ high, attached to the table, preventing the subject from observing an Anscomatic II slide projector. The projector was the source of illumination for a
}

$1^{\circ}$ circular light patch projected on the far wall facing the subject, $3.7 \mathrm{~m}$ distant. A black cloth covered the projector to further reduce stray light in the experimental room. The projector was placed on a wooden swivel container. The experimenter could move the swivel, and thus the spot of light, by pulling on a cord and pulley device attached to it. The location of the spot of light, in angular degrees, was indicated by a metal pointer attached to the swivel device which moved with it and registered the number of degrees of displacement on a protractor. During all conditions in this experiment, the subject's hand was kept out of sight far below eye level.

\section{Design}

A two-factor completely randomized design was employed. The two factors were prism strength $(10,15,20,25$, and $30 \mathrm{D})$ and direction of prism displacement, right and left. There were eight subjects in each of the 10 groups.

\section{Procedure}

All subjects were blindfolded before being led into the experimental room and seated before the apparatus. The blindfold was removed after the reading of instructions and the answering of any relevant questions. The approximate period of dark adaptation was $5 \mathrm{~min}$. The subjects were blindfolded primarily to rule out their knowledge of the room characteristics as an influence in their judgments of the straight-ahead in the dark. There were three successive measurement situations for each of the 10 groups. In each of these, the subjects made four judgments of the apparent straight-ahead by having the experimenter align the projected spot of light ahead of their noses. In all measurement conditions, the subjects were instructed to simply indicate by the word "stop" when they felt that the spot was positioned perpendicularly in front of their noses. These four measurements were counterbalanced across subjects in each group for initial starting position of the projected spot, i.e., whether it began its excursion to the subject's right or left. Successive adjustments alternated sides. For all measurments, the spot was initially positioned at the extreme edge of the subject's field of vision and slowly moved across the field until the subject indicated that the spot was ahead of her nose. The three measurement situations were as follows:

Baseline judgment. The room was dark and the Risley prisms were set to zero. This condition served as a baseline for the subject's judgment of the apparent straight-ahead.

Dark judgment. The room was dark and the prisms were set to the appropriate displacement and base orientation.

Light judgment. A floor light to the left of the subject $(150 \mathrm{~W})$ was turned on. It illuminated the room such that shadows were minimized. The prisms remained set at the same displacement as in the previous condition.

\section{Results}

The first analysis involves the initial judgment of the straight-ahead in the baseline judgment condition. Although none of the experimental manipulations had occurred at this point, it was important that the groups not exhibit differences in their judgments before the prism displacements were encountered. Such was the situation in that neither the Prism Strength, $\mathrm{F}(4,70)=.24$, Prism Orientation, $\mathrm{F}(1,70)$ $=.50$, nor the interaction of these factors, $F(4,70)$ $=.91$, was significant.

The second analysis concerned the measurement of the correction effect. This was determined by subtracting the mean of the subject's judgments in the light judgment condition from that obtained in the 
dark judgment condition. This is the expected direction of the correction effect and was considered the positive direction for later discussion purposes. Again, there was no effect of prism orientation, $F(1,70)=.95$. More importantly, there was no differential effect of prism strength on the magnitude of the correction effect, $F(4,70)=1.00$. Similarly, the interaction of these factors was not significant, $F(4,70)=.16$.

Table 1 contains the mean correction effects for the five prism strength conditions. They range from $3.06^{\circ}$ for the $15-\mathrm{D}$ condition to $4.60^{\circ}$ for the $20-\mathrm{D}$ group. Over all conditions, the mean correction effect was $3.76^{\circ}$. All of these correction effects, as indicated in the table, were significantly different from zero at the .0005 level, as evaluated by twotailed $t$ tests for correlated measures.

The lack of significant difference in magnitude between the correction effects for the various prism strengths cannot be attributed to any "ceiling effect" in the subject's ability to respond to the displacement produced by the prisms. Difference scores were calculated between the subject's initial placement in the dark when the prismatic deviations were first introduced. The latter judgment represents the amount of prismatic deviation that is being responded to or corrected for in centering the spot of light. The correction is always in the direction opposite to the prismatic deviation. These difference scores were (in degrees) $4.07,6.04,9.14,10.18$, and 15.66 for the 10-, 15-, 20-, 25-, and 30-D conditions, respectively. They were significantly different at the .001 level, $F(4,70)=29.03$. These data emphasize how accurate the subjects were in compensating for the prism displacement in the initial judgments in the dark room. It is, in fact, surprising how accurate they were. In all but the 25-D condition, they were within $2 \frac{1 / 2^{\circ}}{}$ of the true straight-ahead.

\section{EXPERIMENT 2}

\section{Method}

\section{Subjects \\ The subjects were 16 female undergraduates selected from the same pool used in Experiment 1.}

\footnotetext{
Design and Procedure

Two groups of eight subjects went through exactly the same procedures as the two 20-D groups of Experiment 1 except for the method used in obtaining judgments. In the present experiment, the subject pointed with the index finger of her right hand rather than instructing the experimenter in positioning a projected spot of light. The subjects's eyes were open and her hand was kept out of sight below a shelf on the same table used in Experiment 1 . The finger was positioned in a brace on top of a slide mechanism. As the finger was moved, a ruler moved with it, revealing the orientation of the subject's finger. Again, four deter-
}

Table 1

Mean Correction Effects (in Degrees)

\begin{tabular}{ccc}
\hline Condition & Mean & SD \\
\hline 10D & $3.14^{*}$ & 2.92 \\
15D & $3.06^{*}$ & 2.72 \\
20D & $4.60^{*}$ & 3.01 \\
25D & $3.44^{*}$ & 3.33 \\
30D & $4.56^{*}$ & 2.92 \\
\hline
\end{tabular}

$* p<.0005$

minations of the straight-ahead were obtained in each of the three measurement situations. The instructions given to the subjects were comparable to those used in Experiment 1. Instead of the subject saying "stop" to control the movement of the spot of light, she was instructed to position her index finder so that it was pointed straight ahead, i.e., along a line perpendicular to her nose. The subject's eyes were open during these judgments.

\section{Results}

As would be expected from the results of the previous experiment, there were no effects of prism-base orientation on the initial baseline judgments of the subjects or on the magnitude of the correction effect. The mean correction effect across both groups was only $.25^{\circ}$ and was not significantly different from zero, $\mathrm{t}(15)=.38$.

\section{DISCUSSION}

There are three factors about the correction effect that have either been reviewed here or presented within the experimental data: (1) The correction effect is constant across prism strength of 10-30 D. (2) It is not associated with aftereffects when passive movement of the indicator spot of light is used. (3) The correction effect does not occur when pointing responses are used to judge the straight-ahead. There seems to be no uncomplicated way to incorporate these facts into the hypothesis of Rock et al. (1966), discussed earlier, in which the correction effect represents a new relationship between eye position and the spatial coordinate of straight-ahead. It would certainly be expected that prism strength would influence such a recalibration process.

Another approach to explaining the correction effect is that of Harris (1974) through the use of his straight-ahead shift hypothesis. The straight-ahead shift is said to occur to the extent that the observer uses environmental information to define the spatial direction, straight ahead, rather than the median plane of his or her body. Viewing through prisms is portrayed as akin to an observer looking down a hallway while standing somewhat turned towards a corner rather than perpendicular to the far wall. When asked to point straight ahead or to position a spot of light to the visual straight-ahead, an observer may 
very well be confused. Straight-ahead can be either perpendicular to the far wall (using environmental information) or towards the corner in the median plane. Harris suggests that observers wearing prisms, or observers in his "thought experiment" in the corridor, may compromise their judgments towards the environmental coordinate while the experimenter assumes they are judging with respect to the median plane. Harris proposes that this can happen even when the concept of the median plane is explained to the observer.

Harris uses the correction effect as his first example of a straight-ahead shift. The shift towards veridicality in the illuminated room portion of the correction effect procedure is said to represent a compromise that develops, in the subject's judgment of the straight-ahead, between the locations indicated by the median plane of his or her body and the prismatically shifted environmental coordinates. The data of the present study do not support this interpretation. If the correction effect represents an instance of the straight-ahead shift, it would be expected to increase with increases in the magnitude of the imposed prismatic deviation. The discrepancy between the subject's median plane and the environmental determinants of the straight-ahead increases coincidentally with the increases in prism strength. In addition, to the extent that there is confusion with regard to the spatial coordinate, straight ahead, it should have shown up in the pointing response as well as in the spot positioning task.

Another approach to explaining the correction effect is to view it as the consequence of sustained potentiation of the extraocular muscles, i.e., as an eye-position aftereffect. Ebenholtz and his associates (e.g., Papp \& Ebenholtz, 1976) have argued that aftereffects in the visual position sense that occur in prism adaptation paradigms can best be explained through the use of a muscle potentiation hypothesis rather than with the traditional recalibration hypotheses of prism adaptation (Epstein, 1975). Basically, they argue that in prismatic viewing the eyes are maintained in a restricted range of asymmetrical convergence positions. This is said to induce potentiation in the extraocular muscles that continues upon removal of the prismatic displacement. This potentiation is a continued innervation in these muscles in the direction induced by the previous prismatic displacement. Papp and Ebenholtz show that sizable aftereffects can be produced by maintaining these lateralized eye positions, without prisms, for as short a time as $30 \mathrm{sec}$. These aftereffects are in the direction opposite to the induced eye position. This direction is the same that would occur if prisms had been used to produce the effect, i.e., in the direction of the prism apex.
Although the present data on potentiation aftereffects are limited to the exposure durations and displacement magnitudes used by Papp and Ebenholtz, their initial findings do offer some promise that the potentiation model may aid in the interpretation of the correction effect. First, it should be recalled that the subjects of the present study showed a shift, in their initial judgments of the straight-ahead in the dark under prism viewing, that was an incomplete correction for the optical displacement. This initial error, averaging about $2.4^{\circ}$, was always in the direction of the prism apex as would be expected if a potentiation effect was occurring.

A second point of contact between the potentiation and correction effect paradigms concerns the extensive measurements of the straight-ahead in the latter case. The eight measurements of the straight-ahead in the present experiment (four each in the dark and in the light) probably took anywhere between 4 and $8 \mathrm{~min}$, depending on the particular subject and her judgment criteria. This time factor was an uncontrolled source of variance in the experiment. It would be expected that potentiation effects, if they should occur, would continue to develop throughout both illumination conditions. The correction effect would, in this case, simply represent an enhancement, in the illuminated room condition, of the potentiation effect established in the dark viewing condition. An important question becomes the asymptotic value of potentiation aftereffects that would be expected from such exposure conditions, durations, and prism strengths. The Papp and Ebenholtz study gives some tentative information, although the uncontrolled exposure durations of the present study have to be recognized. Papp and Ebenholtz obtained a ceiling effect in their aftereffects for a $32^{\circ}$ lateral displacement that is intriguing in its value of $3.52^{\circ}$. This is the average aftereffect over their 2-, 4-, and 8-min exposure duration conditions which were not significantly different in aftereffect magnitude. This value is very similar to the correction effect produced in each of the five prism strength conditions of the present study, even though the magnitude of displacement in the Papp and Ebenholtz study was $15^{\circ}$ greater than the largest used in the present study.

Although Papp and Ebenholtz obtained a generally monotonic relationship between magnitude of induced eye turn and aftereffect for short exposure durations, it is not clear that such a relationship would hold up at longer exposure durations. If it did, the potentiation model would not be able to handle the constancy of the correction effect over the range of prismatic displacements used in the present study. It may be that the asymptotic value of the potentiation effect is reached at longer durations for the smaller prism strengths. In Papp and Ebenholtz's $12^{\circ}$ 
condition, the only one in the range of displacements used in the present study, an average aftereffect of $2.2^{\circ}$ was obtained after only $2 \mathrm{~min}$.

What is clearly indicated is an experiment in which induced eye turns in the $6^{\circ}-17^{\circ}$ range are applied for durations up to $8 \mathrm{~min}$ under both light and dark illumination conditions. Support for an eye-muscle potentiation explanation of the correction effect would be obtained to the extent that a common ceiling for the potentiation aftereffect in the $3^{\circ}-4^{\circ}$ range was found for these induced eye turns.

A final note concerns the lack of a correction effect for the pointing response condition of the present study. To the extent that the correction effect reflects potentiation in the extraocular muscles, only visual positioning effects would be expected. Localizing the arm with respect to the body's median plane would not involve eye posture.

\section{REFERENCES}

EpsteIN, W. Recalibration by pairing: A process of perceptual learning. Perception, 1975, 4, 59-72.
HARRIs, C. S. Beware the straightahead shift: A nonperceptual change in experiments on adaptation to displaced vision. Perception, 1974, 3, 461-476.

Papp, K. P., \& Ebenholtz, S. M. Perceptual consequences of potentiation in the extraocular muscles: An alternative explanation for adaptation to wedge prisms. Journal of Experimental Psychology: Human Perception and Performance, 1976, 2, 457-468.

Rock, I., Goldrerg, J., \& MAck, A. Immediate correction and adaptation based on viewing a prismatically displaced scene. Perception \& Psychophysics, 1966, 1, 351-354.

Wallace, B., Melamed, L. E., \& Cohen, R. R. An analysis of aftereffects in the measurement of the correction effect. Perception \& Psychophysics, 1973, 14, 21-23.

Wallace, B., Melamed, L. E., \& Kaplan, C. Movement and illumination factors in adaptation to prismatic viewing. Perception \& Psychophysics, 1973, 13, 164-168.

Wilkinson, D. A. Visual-motor control loop: A linear system? Journal of Experimental Psychology, 1971, 89, $250-257$.
(Received for publication July 1, 1977; revision accepted November $30,1977$. 